\title{
Transfer Effects and Conditional Learning in Rats With Selective Lesions of Medial Septal/Diagonal Band Cholinergic Neurons
}

\author{
Agnieszka M. Janisiewicz and Mark G. Baxter \\ Harvard University
}

\begin{abstract}
The authors examined visual-spatial conditional learning with automated touchscreen tasks in male Long-Evans rats with selective lesions of medial septal/vertical limb of diagonal band (MS/VDB) cholinergic neurons produced by $192 \mathrm{IgG}$-saporin. Performance on a conditional task, in which 1 of 2 centrally displayed stimuli directed the rat to respond to an illuminated panel on the left or right, depended on training history: Control rats with experience on other visual tasks performed better than MS/VDB-lesioned rats with similar training histories, whereas this effect was reversed in naive rats. This difference appears to reflect transfer effects present in the control rats that are absent in the MS/VDBlesioned rats. These findings may suggest that MS/VDB cholinergic neurons play a particular role in the transfer of behavioral experience and flexibility of application of behavioral rules in memory, rather than a role in conditional learning per se.
\end{abstract}

Cognitive functions of the basal forebrain cholinergic system remain difficult to classify. A program of research using selective ablation of these neurons with immunotoxins, directed at the low-affinity nerve growth factor receptor expressed on the surface of these neurons, has indicated that the basal forebrain is not critically involved in many forms of learning and memory (Baxter \& Chiba, 1999; Baxter \& Murg, 2002). In contrast, these neurons do seem to be required for modulation of attentional processing (Baxter, Gallagher, \& Holland, 1999; Baxter, Holland, \& Gallagher, 1997; Chiba, Bucci, Holland, \& Gallagher, 1995; McGaughy, Dalley, Morrison, Everitt, \& Robbins, 2002; McGaughy, Kaiser, \& Sarter, 1996; Waite, Wardlow, \& Power, 1999), as well as some aspects of perceptual processing (Linster, Garcia, Hasselmo, \& Baxter, 2001) and associative learning and recall (Berger-Sweeney, Stearns, Frick, Beard, \& Baxter, 2000; ValeMartínez, Baxter, \& Eichenbaum, 2002).

More specifically, cholinergic neurons in the medial septum/ vertical limb of the diagonal band (MS/VDB), which provide cholinergic input to the hippocampus and cingulate cortex, appear to be involved in conditional learning (Janisiewicz, Jackson, Firoz, \& Baxter, in press; Ridley, Barefoot, Maclean, Pugh, \& Baker, 1999). Our initial study of conditional learning in rats with cholinergic MS/VDB lesions examined the effect of these lesions on an environment-spatial conditional learning task, in which two different sets of local environmental cues signaled the location of

Agnieszka M. Janisiewicz and Mark G. Baxter, Department of Psychology, Harvard University.

This work was supported in part by the Harvard College Research Program. Mark G. Baxter is an Alfred P. Sloan Research Fellow. We thank Silas Wang for programming, Matthew Ian Tomey for assistance in data collection in Experiment 2, Steve Kim for assistance with immunohistochemistry, and Tim Bussey and Eve De Rosa for helpful comments on the manuscript.

Correspondence concerning this article should be addressed to Mark G. Baxter, Department of Psychology, Harvard University, 906 William James Hall, 33 Kirkland Street, Cambridge, Massachusetts 02138. E-mail: mbaxter@wjh.harvard.edu a reward buried in one of eight locations (Janisiewicz et al., in press). Rats, like monkeys, with damage to this system could perform conditional discrimination tasks if pretrained separately on one of the different components of the conditional problem (Janisiewicz et al., in press; Ridley \& Baker, 1997). This implies that the involvement of the septohippocampal system in conditional learning reflects a particular kind of information processing or encoding rather than storage of conditional associations (Ridley \& Baker, 1997).

In the present study, we sought to examine the generality of MS/VDB cholinergic lesion effects on conditional learning. We used the somewhat impoverished environment produced by the touchscreen apparatus (Bussey, Muir, Everitt, \& Robbins, 1997; Bussey, Muir, \& Robbins, 1994) to present two-dimensional visual discrimination problems to rats. Rats received injections of the selective cholinergic immunotoxin 192 IgG-saporin into the MS/ VDB (Baxter, Bucci, Gorman, Wiley, \& Gallagher, 1995) or a control surgery and then began training on the discrimination learning tasks. In particular, we tested rats on a conditional task that is impaired, in terms of both new learning and retention, by cholinergic MS/VDB lesions in monkeys (Ridley et al., 1999). In Experiment 1, rats were trained on a simple visual discrimination (see Figure 1) followed by the Ridley double-cue visual-spatial conditional discrimination. In this double-cue visual-spatial conditional discrimination task, one of two different pairs of identical visual stimuli is presented to the animal (AA and $\mathrm{BB}$ ). If stimuli AA are presented, the animal is reinforced for choosing the stimulus on the left; if stimuli BB are presented, the animal is reinforced for choosing the stimulus on the right. By using a behavioral task that was formally identical to that used to test conditional learning in monkeys, we hoped to ascertain whether conditional learning impairments are a general consequence of damage to septal cholinergic neurons.

As a follow-up, we tested rats' performance in a second centralcue visual-spatial conditional problem, in which a centrally located visual stimulus ( $\mathrm{X}$ or $\mathrm{Y}$ ) directs the rat to make a response to the left or right (Bussey, Muir, Everitt, \& Robbins, 1996; Bussey et al., 1997; Muir, Bussey, Everitt, \& Robbins, 1996). Although 


\section{A: Simple Visual Discrimination}
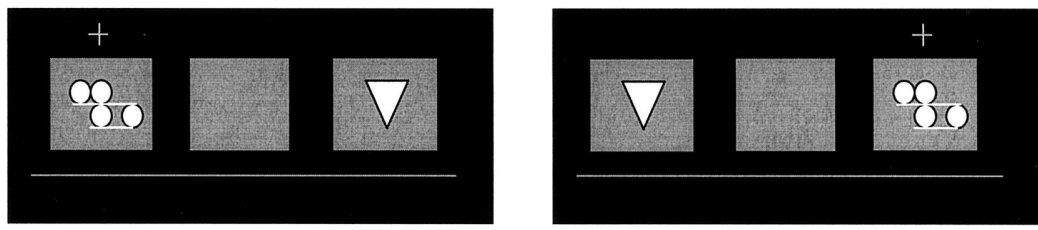

\section{B: Double-Cue Visual-Spatial Conditional}
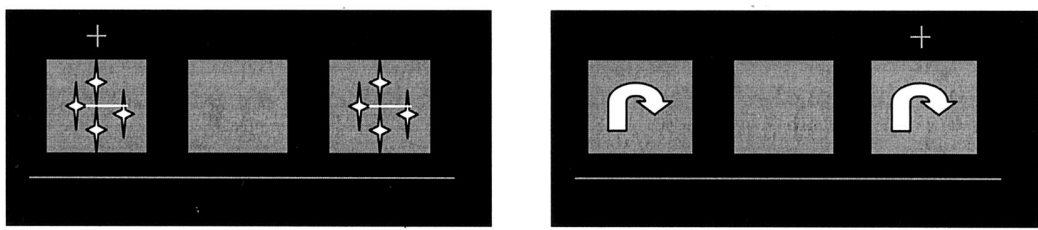

\section{C: Central-Cue Visual-Spatial Conditional}
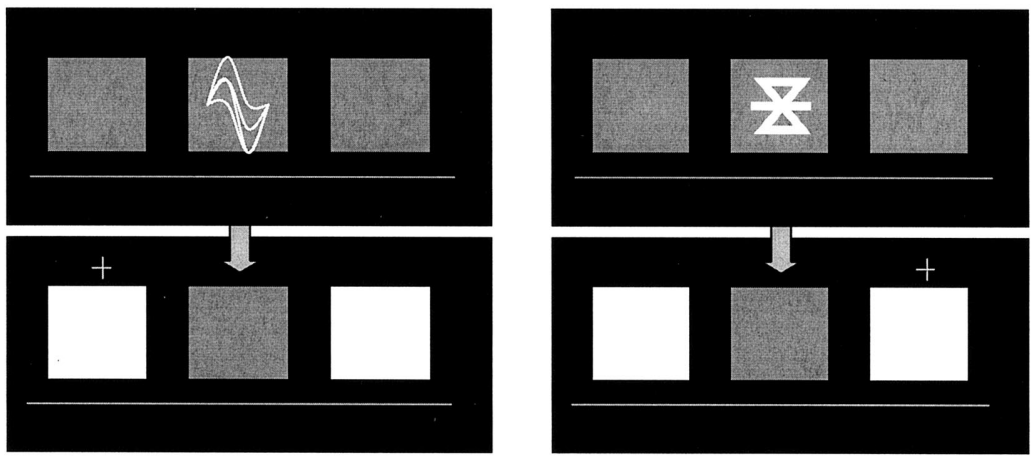

Figure 1. Representations of the touchscreen stimuli for each of the three discriminations. The black rectangles represent the mask covering the touchscreen; the gray rectangles represent the viewing windows. In reality, the stimuli appeared on a black background on the video display of the touchscreen. The gray line below the viewing windows represents the shelf attached to the touchscreen mask. For each discrimination, the two columns represent the two possible trial types, which were pseudorandomly intermixed. A: Simple visual discrimination (Experiment 1A). On each trial, two different stimuli were presented, one in each of the left and right viewing windows. The same stimulus was correct (indicated by a gray plus sign) regardless of whether it appeared on the left or right. B: Double-cue visual-spatial conditional discrimination (Experiment 1B). One of two new pairs of identical stimuli could appear in the left and right viewing windows. Depending on which stimulus pair appeared, either the left or right member of the pair was correct. C: Central-cue visual-spatial conditional discrimination (Experiments 1C and 2). One of two new stimuli could appear in the central viewing window. The rat was required to touch the stimulus in the central window, whereupon it disappeared and the two side windows were illuminated. Depending on the identity of the stimulus that had been in the center, a touch to either the left or right window was correct. The stimuli presented in the figure resemble those presented to the rats.

this latter task also requires the use of visual information to emit a spatially directed response, lesions of the fornix or MS/VDB in rats do not impair performance on this task (Bussey, Duck, Muir, \& Aggleton, 2000; Marston, Everitt, \& Robbins, 1993). Even though this seems paradoxical, it is possible that this task engages different conditional learning mechanisms because the spatial response is directed at a location different from the visual stimuli, unlike the double-cue task in which the spatial response is directed at one of the visual stimuli. Nevertheless, if rats with lesions of the MS/VDB do indeed have a generalized deficit in conditional learning like monkeys, one would predict an exacerbated behavioral deficit on this task. Because we found an unexpected effect of MS/VDB lesions on performance of the central-cue task after rats had been tested on the other two tasks, we ran another experiment
(Experiment 2) examining effects of these lesions on the centralcue task without previous testing on the other discriminations. These types of experiments provide insight into the conditions under which intact basal forebrain cholinergic neurons are required for normal learning and memory function.

\section{Method}

\section{Subjects}

Twenty-four male Long-Evans rats from Taconic breeding colony (Germantown, NY) weighed 300-350 g at the beginning of the experiment and were housed at $22{ }^{\circ} \mathrm{C}$ under a 12 -hr light-dark cycle. They had free access to water and food until 2 weeks postsurgery. At this point, they were placed on a restricted feeding schedule to reduce them to $85 \%$ of their free-feeding 
baseline weight. Each rat was individually housed and handled daily before behavioral testing. Their treatment complied with federal, state, and local guidelines. The animal facilities at Harvard University are fully accredited by the Association for Assessment and Accreditation of Laboratory Animal Care, and all animal protocols were approved by the Harvard University Standing Committee on the Use of Animals in Research and Teaching.

\section{Surgery}

Rats were divided into two groups: those receiving cholinergic medial septal lesions (MS/VDB, $n=12$ ) and surgical controls (CONT, $n=12$ ). Prior to surgery, each rat was deeply anaesthetized by an intramuscular injection of a ketamine-xylazine mixture $(80 \mathrm{mg} / \mathrm{kg}$ ketamine and $5 \mathrm{mg} / \mathrm{kg}$ xylazine; Phoenix Pharmaceuticals, St. Joseph, MO). They were also given atropine $(0.25-0.3 \mathrm{ml}$ of a $54 \mathrm{mg} / \mathrm{ml}$ solution intraperitoneally; Phoenix Pharmaceuticals, St. Joseph, MO) to reduce salivation. Additional ketamine $(10 \mathrm{mg} \mathrm{im})$ was given every $45 \mathrm{~min}$ to maintain anesthesia. The rat's head was then shaved and placed in a stereotaxic headholder (Kopf Instruments, Tujunga, CA), with the incisor bar $3.3 \mathrm{~mm}$ below the interaural line. The skin overlying the skull was disinfected with povidoneiodine solution (Betadine). The scalp was cut minimally with a single incision to expose the skull. Drill holes large enough to accommodate a 28-gauge needle were made at $+0.45 \mathrm{~mm}$ anterior and $\pm 0.6 \mathrm{~mm}$ lateral to bregma (coordinates taken from Baxter et al., 1995). In each of these two sites, injections of $192 \mathrm{IgG}$-saporin $(0.12 \mu \mathrm{g} / \mu \mathrm{l}$; Chemicon, Temecula, CA) for lesion surgeries or sterile phosphate-buffered saline for control surgeries were placed at two positions: $0.3 \mu \mathrm{l}$ at $-7.8 \mathrm{~mm}$ and $0.2 \mu \mathrm{l}$ at $-6.2 \mathrm{~mm}$ relative to the skull surface measured at bregma (flow rate 0.05 $\mu \mathrm{l} / \mathrm{min}$ for both). The needle was left in place for an additional $9 \mathrm{~min}$ and 6 min, respectively, after the completion of each injection to allow the toxin to diffuse from the injection site. Afterward, the scalp was sutured with Vicryl 3-0 sutures (Ethicon, Somerville, NJ), and an antibiotic ointment with pain reliever was applied to the wound. Rats were given intraperitoneal injections of electrolyte fluids $(1 \mathrm{cc})$ for rehydration and intramuscular injections of Banamine (flunixin meglumine, $0.5 \mathrm{mg}$ in 0.1 $\mathrm{ml}$; Schering-Plough Animal Health, Union, NJ) as an analgesic. In addition, Banamine $(0.5 \mathrm{mg} \mathrm{im})$ was given for two consecutive days postoperatively to aid in recovery, and food was saturated with water to help the animals rehydrate. A minimum of 2 weeks was given for full recovery.

\section{Apparatus}

The test apparatus was constructed according to the specifications of Bussey and colleagues (Bussey et al., 1994, 1997). A clear Plexiglas box $(0.50 \mathrm{~m} \times 0.25 \mathrm{~m} \times 0.50 \mathrm{~m})$ with a metal floor was fitted with a touch-sensitive computer screen on one end (Carroll Touch, Round Rock, TX). On the opposite end, a weight-sensitive floor panel was located in front of a food magazine with an infrared detector, connected to an automatic pellet dispenser. Furthermore, the front of the computer screen was covered with a Plexiglas mask that contained three open viewing windows $(8 \times 9 \mathrm{~cm})$ over a spring-mounted shelf. The shelf was $9.5 \mathrm{~cm}$ above the floor of the test apparatus. This mask served to encourage the rat to rear and touch the computer screen in three distinct areas. The box was also equipped with a houselight and tone generator in the ceiling and a light above the food magazine. The touchscreen and other electronic components were controlled by custom-written programs in Visual Basic. These programs placed all elements of the testing program under computer control. The general test sequence was programmed so that the rat was required to press the floor panel in front of the food magazine to begin a trial and touch the image designated as correct on the computer screen to receive a reward. If the rat chose correctly, then the tone generator was activated for $1 \mathrm{~s}$, the light over the food magazine was illuminated, and a single 45-mg pellet (Research Diets, New Brunswick, NJ) was delivered to the feeder. Three seconds after the rat put his head in the feeder to retrieve the reward pellet (detected by the infrared detector on the food magazine), another trial could be initiated by standing on the floor panel. If the rat chose incorrectly, no food pellet was released and the houselight was extinguished for $5 \mathrm{~s}$. After this interval, the houselight was reilluminated and standing on the floor plate would initiate a new trial. The entire apparatus was enclosed with a wooden shell $(0.75 \mathrm{~m} \times 0.50 \mathrm{~m} \times 0.75 \mathrm{~m})$ open at one end to accommodate the computer monitor and touchscreen. A door with a clear red Plexiglas panel permitted covert viewing of the rat during testing sessions.

\section{Pretraining}

All rats were initially habituated 1 day for $30 \mathrm{~min}$ in their training box. During this period, food pellets were placed in the magazine and on the shelf below the viewing windows in the mask covering the touchscreen. The rats were then given 2 days ( 50 trials per day) of pretraining designed so that rats would acquire an association between the magazine light, the tone, and the food reward release. On each pretraining trial, either the right or left panel would be illuminated for $10 \mathrm{~s}$ by displaying a white rectangle behind that panel on the computer screen. At the end of the 10-s interval, the rectangle disappeared, the tone was activated for $1 \mathrm{~s}$, the light over the food magazine was illuminated, and a food pellet was delivered. Next, the rats were explicitly trained to respond to the illumination of the screen with a nose poke. In this program, either the left or right panel would be illuminated. To make a correct choice, the rat had to nose poke into the illuminated panel to be rewarded. If the rat made an incorrect choice by nose poking in any other panel, the stimulus disappeared and the houselight was extinguished for $5 \mathrm{~s}$. Training in this phase was continued for 50 trials per day until the rats reached criterion, which consisted of finishing the session in less than $30 \mathrm{~min}$ and obtaining $85 \%$ correct during two consecutive sessions. In all of the programs, if a rat exhibited a side bias at the end of 1 day of testing (responding to one side on $70 \%$ or more of trials), correction trials were given on the following day to extinguish the side bias. During correction trials, incorrect trials were repeated until a correct response was made. These trials did not count toward the session total of 50 or 100 trials (100 trials per day were given in subsequent training). Typically, only 1 day of correction trials was required to correct a side bias. Each of the four available boxes was cleaned between rats with a $50 \%$ water-ethanol solution.

\section{Experiment 1A: Simple Visual Discrimination Behavioral Testing}

After reaching criterion on shaping, one group of rats (CONT, $n=6$; MS/VDB, $n=6$ ) began a simple one-pair visual discrimination task. In this task, a pair of images was displayed at the initiation of a trial, one in each of the left and right viewing panels. The images were white shapes of varying complexity on a black background (see Figure 1A). In this task, responses to one image of the pair were always reinforced regardless of its location $(\mathrm{S}+)$, whereas the other was never reinforced. The left-right position of $\mathrm{S}+$ varied pseudorandomly from trial to trial. No images appeared in the central panel in this task. Rats received a daily test session of 100 trials per day until they reached criterion, completing all 100 trials in less than $1 \mathrm{hr}$ and obtaining at least $85 \%$ correct during two consecutive sessions. This definition of criterion applies to all subsequent experiments.

\section{Experiment 1B: Double-Cue Visual-Spatial Conditional Discrimination Behavioral Testing}

After completion of Experiment 1A, the same rats (CONT, $n=6$; MS/VDB, $n=6$ ) went onto a double-cue visual-spatial conditional task In this task, the same image was presented in duplicate in both the left and right panels. These two new pairs of images (AA and $\mathrm{BB}$ ) were presented in a pseudorandom order on the two outer panels (see Figure 1B). When pair AA appeared, the rat would be reinforced for choosing the left A, 
whereas when BB appeared, the rat would be reinforced for choosing the right $\mathrm{B}$. One hundred trials per day were given until criterion was achieved.

\section{Experiments 1C and 2: Central-Cue Visual-Spatial Conditional Discrimination Behavioral Testing}

After completion of Experiment 1B, the same rats (CONT, $n=6$; $\mathrm{MS} / \mathrm{VDB}, n=6$ ) were tested in the central-cue visual-spatial conditional task (Experiment 1C). A second group of rats (CONT, $n=6$; MS/VDB, $n=6$ ) were tested on this immediately after completion of pretraining without undergoing any other discrimination tasks first (Experiment 2). In this task, one of two new stimuli (either $\mathrm{X}$ or $\mathrm{Y}$ ) was presented in the central panel at the beginning of a trial. The rat was required to touch the central panel, at which time the stimulus in the central panel disappeared and the two side panels were both illuminated with white rectangles. The rat then had to touch either the left or right white panel, depending on which object was just presented in the middle, to receive reward. For object $\mathrm{X}$, the left panel was correct; for object $\mathrm{Y}$, the right panel was correct (see Figure 1C). Again, rats were given 100 trials per day until they reached criterion. Testing was discontinued for rats that failed to reach criterion within 50 sessions.

\section{Histological Analysis}

Rats were given a lethal dose of Nembutal $(100 \mathrm{mg} / \mathrm{kg}$; Abbott Laboratories, North Chicago, IL). After induction of terminal anesthesia, the rat was transcardially perfused with $0.9 \%$ saline for $5 \mathrm{~min}$, followed by $4 \%$ freshly depolymerized paraformaldehyde in $0.1 \mathrm{M}$ phosphate buffer for 20 min, both at a flow rate of $18 \mathrm{ml} / \mathrm{min}$. The brain was then extracted and immersed in $4 \%$ paraformaldehyde for another $2 \mathrm{hr}$. Afterward, it was immersed in $20 \%$ sucrose solution for 3 days.

After 3 days in sucrose, $60 \mu \mathrm{m}$ coronal sections through the MS/VDB were taken on a freezing-sliding microtome and stored in $0.1 \mathrm{M}$ phosphatebuffered saline until histology. All cases were processed for choline acetyltransferase (ChAT) immunohistochemistry to detect the presence or absence of cholinergic neurons in the MS/VDB. A limited number of cases (MS/VDB, $n=2$; CONT, $n=2$ ) were also processed for parvalbumin immunohistochemistry to confirm the selectivity of the immunotoxic lesion. We did not deem it necessary to perform the second stain on all cases because in our laboratory, we routinely find that immunotoxic lesions do not damage parvalbumin immunoreactive MS/VDB neurons. Furthermore, any nonspecific damage or tissue necrosis that might be observed could be detected in the ChAT-immunostained sections. Immunohistochemical procedures used goat polyclonal anti-ChAT (Chemicon AB144P, Temecula, CA) or mouse monoclonal antiparvalbumin (Sigma Chemical, St. Louis, MO) primary antibodies and followed standard avidin-biotin complex staining procedures (e.g., Berger-Sweeney et al., 2000; Cahill \& Baxter, 2001).

\section{Statistical Analysis}

For each task, repeated measures analysis of variance (ANOVA) was applied to the number of errors (on noncorrection trials) made on each day of testing. Because rats reached criterion at different rates, these analyses only extended to the point that the first rat in each experiment reached criterion. These analyses served to examine effects of lesion on initial acquisition of the behavioral tasks. An additional comparison of sessions to criterion permitted comparison of the amount of time required for each rat to master each discrimination problem. We also considered whether more detailed analysis of intermediate criterion levels (e.g., sessions to move from chance performance to statistically above chance) might reveal effects on different phases of learning (e.g., Bussey et al., 1997). Because not all of the rats in Experiments 1C and 2 reached criterion on the central-cue visual-spatial discrimination, we were only able to analyze the sessions required to move from chance performance to above chance ( $61 \%$ correct).
For this latter measure, the same criterion was applied as for completion of the discrimination problems, except for the level of performance required (two consecutive sessions at or above $61 \%$ correct).

\section{Results}

\section{Histological Analysis}

All MS/VDB-lesioned animals included in this study had complete MS/VDB cholinergic lesions as verified by ChAT immunostaining (compare staining in control rats [see Figure 2A] with staining in MS/VDB-lesioned rats [see Figure 2B]). Neither control nor lesion rats had damage to adjacent brain areas containing cholinergic neurons, such as the caudate putamen. No differences were noted in extent of lesions in rats between the two experiments. Furthermore, these lesions were identical in extent to those in previous studies from our laboratory in which quantitative neurochemical assays have confirmed the extent of cholinergic enzyme depletion from hippocampus as $80 \%$ or greater (e.g., Berger-Sweeney et al., 2000; Cahill \& Baxter, 2001). Staining for parvalbumin immunoreactive (GABAergic; Freund, 1989) neurons in the MS/VDB was indistinguishable between control (see Figure 2C) and MS/VDB-lesioned (see Figure 2D) rats. This verifies the cholinergic specificity of 192 IgG-saporin. One MS/ VDB-lesioned rat in Experiment 2 had only limited depletion of cholinergic neurons, so this rat was excluded from the study, bringing the number of MS/VDB-lesioned rats in that experiment to 5 .

\section{Experiment 1A: Simple Visual Discrimination}

No significant difference in acquisition of the simple one-pair discrimination was seen between control and MS/VDB-lesioned rats (see Figure 3A). Analysis of acquisition rate was done for Sessions 1 through 6 because the first rat finished on Session 6 and therefore acquisition data were available for all rats only for the first 6 sessions of testing. A two-way ANOVA, with lesion as a between-subjects factor and session as a within-subject factor, indicated a significant effect of session, $F(5,50)=19.70, p<$ .0005 , but no significant effect of lesion, $F(1,10)=0.35, p=.57$, or Session $\times$ Lesion interaction, $F(5,50)=1.11, p=.37$. There was also no lesion effect on the number of sessions it took each group to reach criterion, $F(1,10)=1.10, p=.32$ (see Figure $3 \mathrm{~B}$ )

\section{Experiment 1B: Double-Cue Visual-Spatial Conditional Discrimination}

No significant difference in acquisition was seen between control and MS/VDB-lesioned rats on the double-cue conditional visual-spatial discrimination (see Figure 4A). Analysis of acquisition rate was done for Sessions 1 through 10 because the first rat finished on Session 10 and therefore acquisition data were available for all rats only for the first 10 sessions of testing. A two-way ANOVA, with lesion as a between-subjects factor and session as a within-subject factor, revealed a significant effect of session, $F(9,90)=20.15, p<.0005$, but no significant effect of lesion, $F(1,10)=0.32, p=.59$, or Session $\times$ Lesion interaction, $F(9$, $90)=0.64, p=.76$. There was also no lesion effect on the number of sessions it took each group to reach criterion, $F(1,10)=0.67$ $p=.43$ (see Figure 4B). 

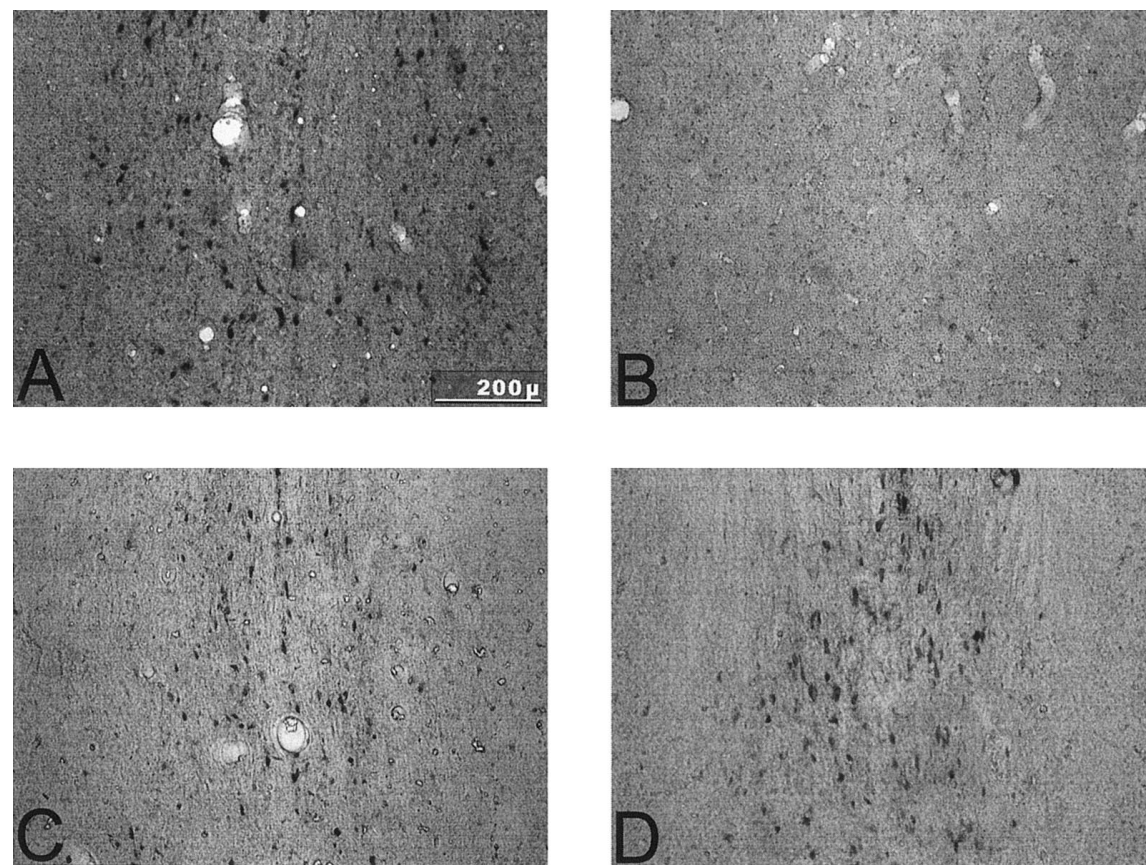

Figure 2. Immunohistochemistry for choline acetyltransferase (A, B) and parvalbumin (C, D) in control (A, C) and medial septal/vertical limb of diagonal band (MS/VDB)-lesioned (B, D) rats. The MS/VDB is devoid of cholinergic neurons in 192 IgG-saporin lesioned rats (B) compared with control rats (A). Parvalbumin immunostaining is intact in the MS/VDB of lesioned rats (D), supporting the specificity of $192 \mathrm{IgG}$-saporin lesions for cholinergic neurons. The scale bar in A applies to all panels.

To examine whether rats found this problem more difficult to learn than a simple discrimination, which would suggest that they were using a different learning strategy, we submitted lesion as a between-subjects factor and experiment $(1 \mathrm{~A}$ or $1 \mathrm{~B})$ as a withinsubject factor to a two-way ANOVA on sessions to criterion. The analysis revealed a main effect of experiment, $F(1,10)=16.29$, $p=.002$, but no effect of lesion or Lesion $\times$ Experiment interaction, $F_{\mathrm{s}}(1,10)<1.07, p \mathrm{~s}>.33$. The double-cue visual-spatial task was more difficult, with an overall mean (control and MS/ VDB-lesioned rats) of 10.17 sessions to criterion on the simple visual discrimination and a mean of 14.25 sessions to criterion on the double-cue task.
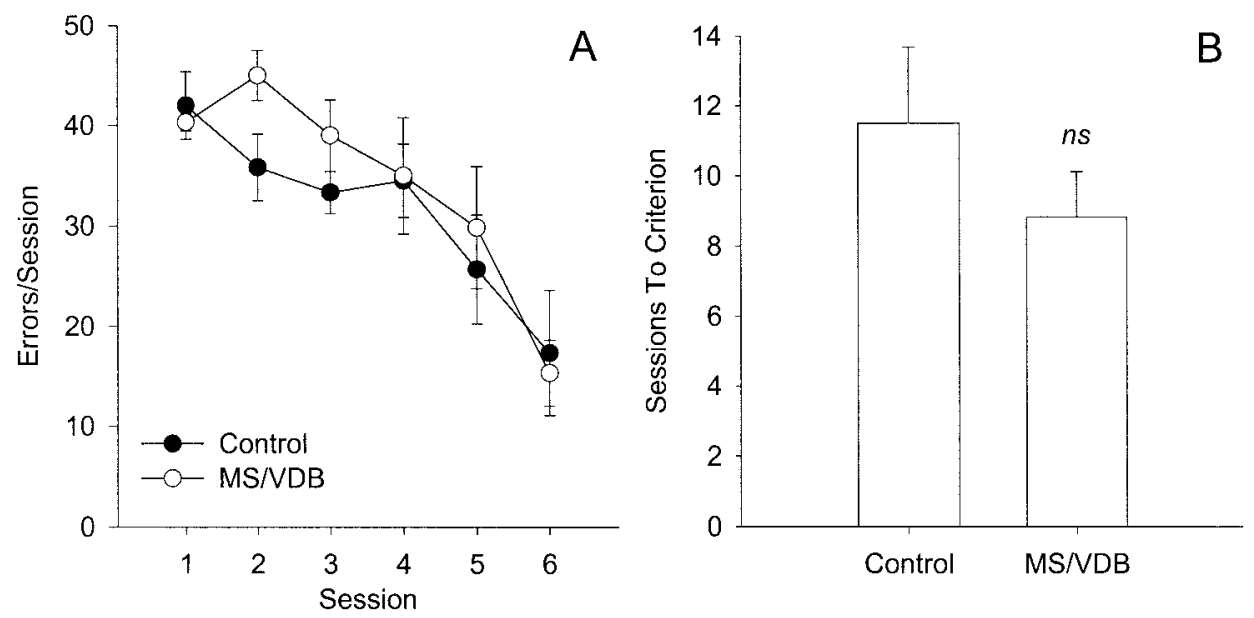

Figure 3. Experiment 1A: Simple visual discrimination. A: Mean errors per session for the first 6 sessions of the simple visual discrimination. Analyses of learning curves were truncated at Session 6, because this is the first session in which any rat completed a criterion run. There is no effect of lesion on learning rate or task performance. B: Mean sessions to criterion in acquisition of the simple visual discrimination. Error bars represent \pm 1 SEM. MS/VDB = medial septum/vertical limb of diagonal band. There is no effect of lesion on sessions to criterion for the simple visual discrimination. 

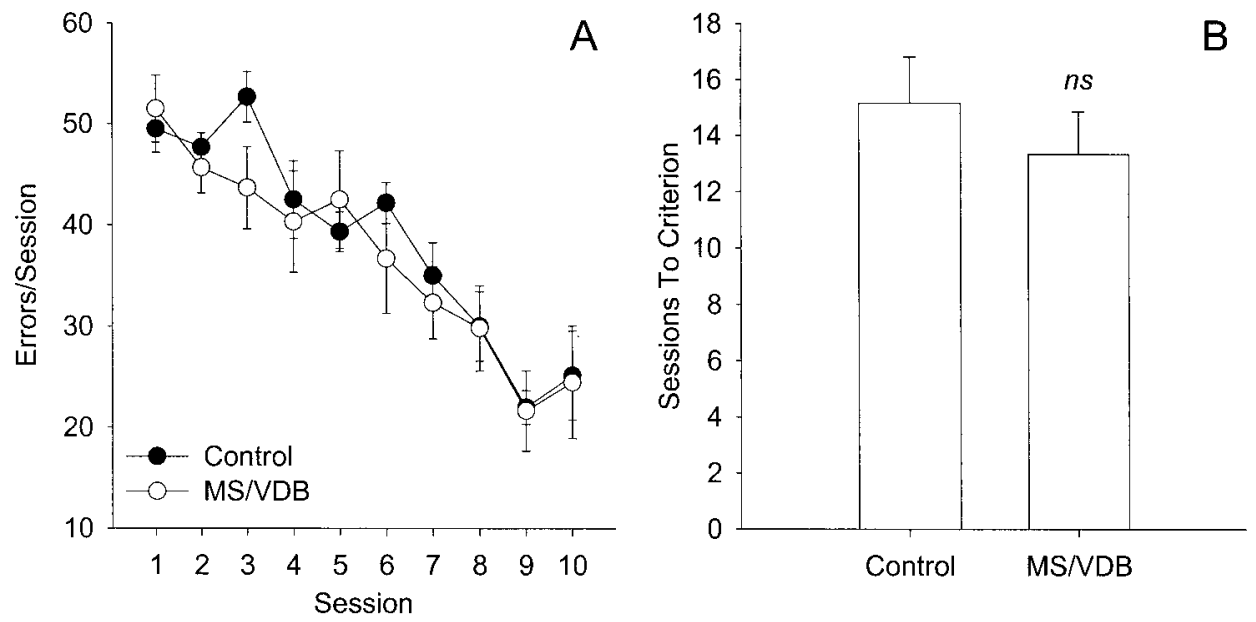

Figure 4. Experiment 1B: Double-cue visual-spatial conditional discrimination. A: Mean errors per session for the first 10 sessions of the double-cue visual-spatial conditional discrimination. Analyses of learning curves were truncated at Session 10, because this is the first session in which any rat completed a criterion run. There is no effect of lesion on learning rate or task performance. B: Mean sessions to criterion in acquisition of the double-cue visual-spatial conditional discrimination. Error bars represent $\pm 1 \quad S E M$. MS/VDB $=$ medial septum/vertical limb of diagonal band.

\section{Experiment 1C: Central-Cue Visual-Spatial Conditional Discrimination}

In contrast to Experiments $1 \mathrm{~A}$ and $1 \mathrm{~B}$, a significant MS/VDBlesion impairment was observed on acquisition of the central-cue visual-spatial conditional discrimination. MS/VDB-lesioned rats performed this discrimination task more poorly than controls. Acquisition data were analyzed with a two-way ANOVA, with lesion as a between-subjects factor and session as a within-subject factor, for the sessions in which data were available for all rats tested (Sessions 1-29; Session 29 was the earliest session in which a rat reached criterion). This analysis revealed a significant main effect of session, $F(28,280)=21.32, p<.0005$, as well as a significant main effect of lesion, $F(1,10)=10.74, p=.008$ (see Figure $5 \mathrm{~A})$. However, the Session $\times$ Lesion interaction was not significant, $F(28,280)=1.03, p=.42$. There was a trend for control rats to complete the task in fewer sessions to criterion relative to $\mathrm{MS} / \mathrm{VDB}$-lesioned rats, although this did not reach significance, $t(10)=1.75, p=.11$ (see Figure $5 \mathrm{~B}$ ). A similar trend was evident in the sessions required to reach an above-chance $(61 \%)$ criterion $(M \pm S E M ; \mathrm{CONT}=7.00 \pm 1.41, \mathrm{MS} / \mathrm{VDB}=$
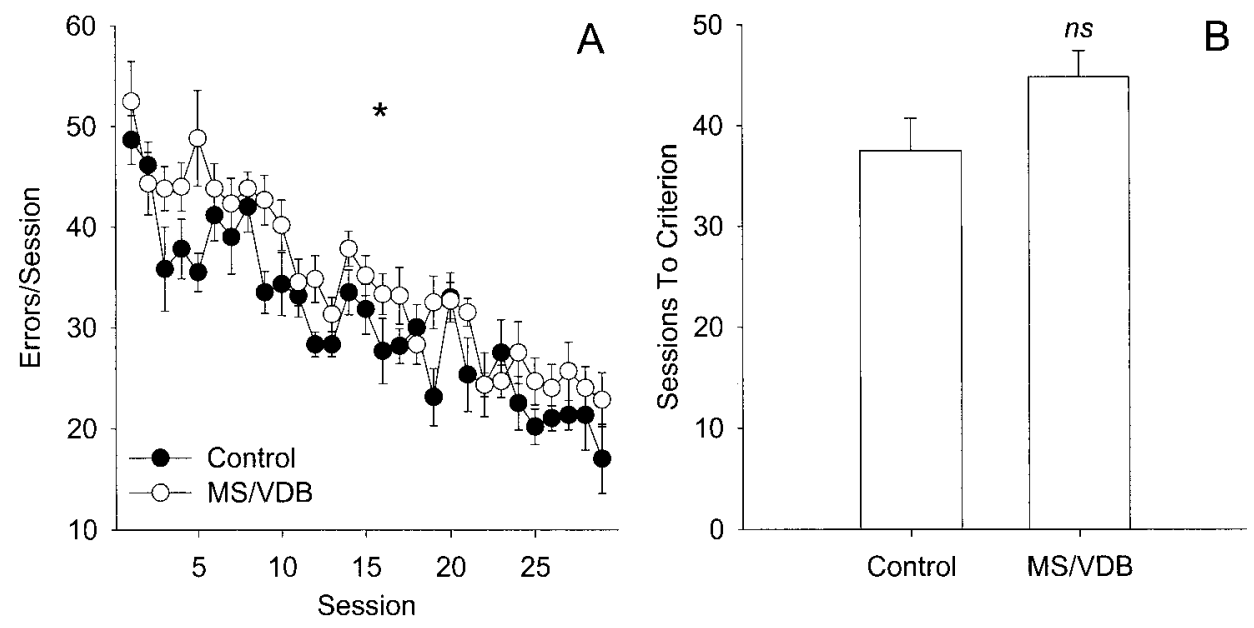

Figure 5. Experiment 1C: Central-cue visual-spatial conditional discrimination. A: Mean errors per session for the first 29 sessions of the central-cue visual-spatial conditional discrimination. Analyses of learning curves were truncated at Session 29, because this is the first session in which any rat completed a criterion run. There is a significant main effect $(p<.05)$ of lesion on performance in the task, with controls performing better than MS/VDB-lesioned rats (overall effect indicated by the asterisk). B: Mean sessions to criterion in acquisition of the central-cue visual-spatial conditional discrimination. Error bars represent $\pm 1 S E M$. MS/VDB $=$ medial septum/vertical limb of diagonal band. There is no effect of lesion on sessions to criterion for this problem. 
$10.33 \pm 0.96), t(10)=1.95, p=.079$. Taken together, these data suggest that MS/VDB-lesioned rats that completed Experiments $1 \mathrm{~A}$ and $1 \mathrm{~B}$ were mildly impaired relative to controls in their subsequent performance of the central-cue visual-spatial conditional discrimination task.

\section{Experiment 2: Central-Cue Visual-Spatial Conditional Discrimination Without Training on Other Discrimination Tasks}

Because we found an unexpected impairment in the central-cue visual-spatial conditional discrimination task in rats with MS/ VDB cholinergic lesions, we considered that the experience of the rats with other discrimination tasks may have affected their performance on the central-cue task. Consequently, we tested a second group of rats on the central-cue task immediately after they finished pretraining. In contrast to the results of Experiment $1 \mathrm{C}$, rats that did not have experience on the other touchscreen programs prior to the central-cue visual-spatial conditional discrimination task were unimpaired in acquiring the central-cue task and moreover were facilitated relative to controls. Acquisition data were analyzed with a two-way ANOVA, with lesion as a betweensubjects factor and session as a within-subject factor, for the sessions in which data were available for all rats tested (Sessions 1-17; Session 17 was the earliest session in which a rat reached criterion). This analysis (see Figure 6A) revealed an overall significant effect of session, $F(16,144)=11.94, p<.0005$, but no overall effect of lesion, $F(1,9)=2.28, p=.17$, and a trend toward a Session $\times$ Lesion interaction, $F(16,144)=1.57, p=.085$. However, analysis of number of sessions to criterion revealed a significant difference between control and MS/VDB-lesioned rats, $F(1,9)=26.93, p=.001$ (see Figure 6B). None of the control rats attained criterion before being stopped at 50 sessions of training, whereas all of the MS/VDB-lesioned rats hit criterion prior to surpassing the maximum number of sessions. There was no difference between control and MS/VDB-lesioned rats in the number of sessions taken to reach an above-chance $(61 \%)$ criterion $(M \pm$ SEM; CONT $=17.33 \pm 6.25, \mathrm{MS} / \mathrm{VDB}=10.2 \pm 2.65), t(9)=$ $0.98, p=.355$. This suggests that learning was more rapid in MS/VDB-lesioned rats in Experiment $2 \mathrm{C}$ after above-chance performance levels were reached, although this analysis could not be conducted explicitly because not all of the rats reached $85 \%$ performance criterion.

We also compared performance on the central-cue visualspatial conditional discrimination task between rats with (Experiment 1C) and without (Experiment 2) training on additional touchscreen discrimination tasks to further understand the origin of the lesion effects. This direct comparison revealed that rats with MS/ VDB lesions performed comparably, regardless of whether they had previous experience with other discrimination problems in the touchscreens, whereas control rats performed much better on the central-cue visual-spatial conditional discrimination task if they had been pretrained on other touchscreen tasks (see Figures 7A and 7B). First, a repeated-measures ANOVA with two betweensubjects factors (lesion and pretraining) and one within-subject factor (session) was conducted on initial acquisition for the sessions for which data were available for all rats (Sessions 1-17, the first day in which a rat in either Experiment $1 \mathrm{C}$ or 2 reached criterion). This analysis revealed a significant Lesion $\times$ Pretraining interaction, $F(1,19)=7.78, p=.01$; main effects of lesion and pretraining were not significant, $F \mathrm{~s}(1,19)<1.00, p \mathrm{~s}<.40$. A main effect of session was significant, $F(16,304)=22.21, p<$ .0005 , but interactions of this effect with effects of lesion, pretraining, or Lesion $\times$ Pretraining were not significant, $F \mathrm{~s}(16$, $304)<1.39, p s>$.14. A similar analysis for sessions to criterion
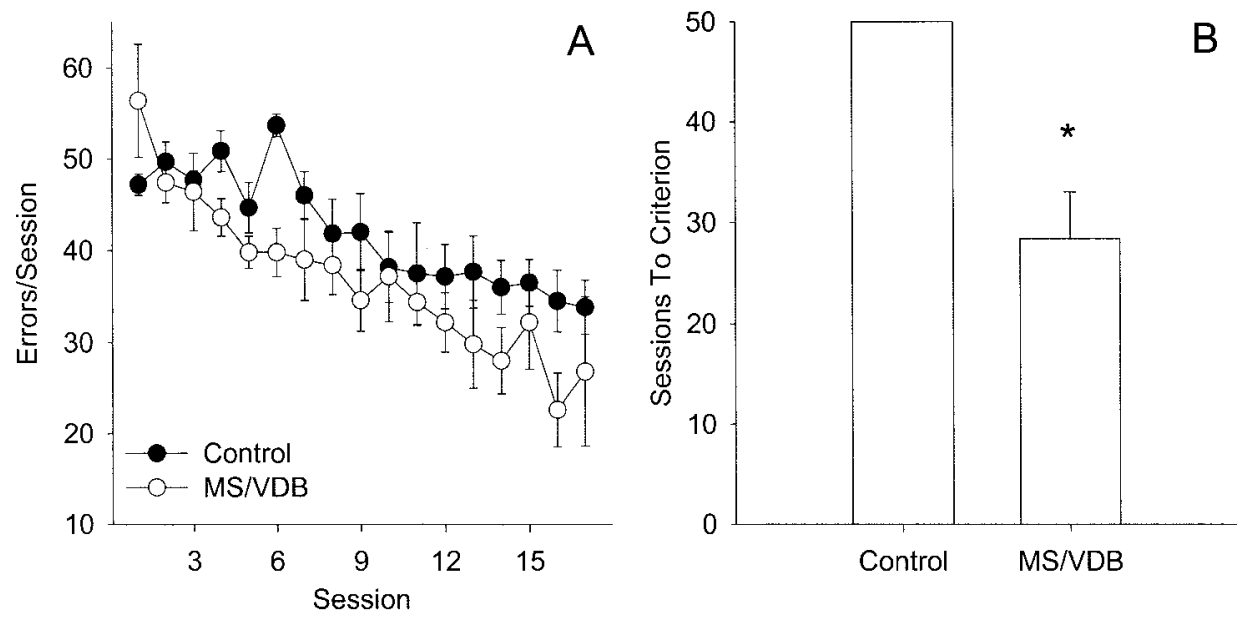

Figure 6. Experiment 2: Central-cue visual-spatial conditional discrimination with no prior training on other visual discriminations. A: Mean errors per session for the first 17 sessions of the central-cue visual-spatial conditional discrimination for rats without prior training on a simple visual discrimination or on the double-cue visual-spatial conditional discrimination. Analyses of learning curves were truncated at Session 17, because this is the first session in which any rat completed a criterion run. There is no effect of lesion on learning rate or task performance. B: Mean sessions to criterion in acquisition of the central-cue visual-spatial conditional discrimination in non-pretrained rats. There is a significant effect $(p<.05)$ of lesion on sessions to criterion for this problem, with MS/VDB-lesioned rats attaining criterion faster than controls (overall effect indicated by the asterisk). Error bars represent $\pm 1 S E M$. MS/VDB $=$ medial septum/vertical limb of diagonal band. 

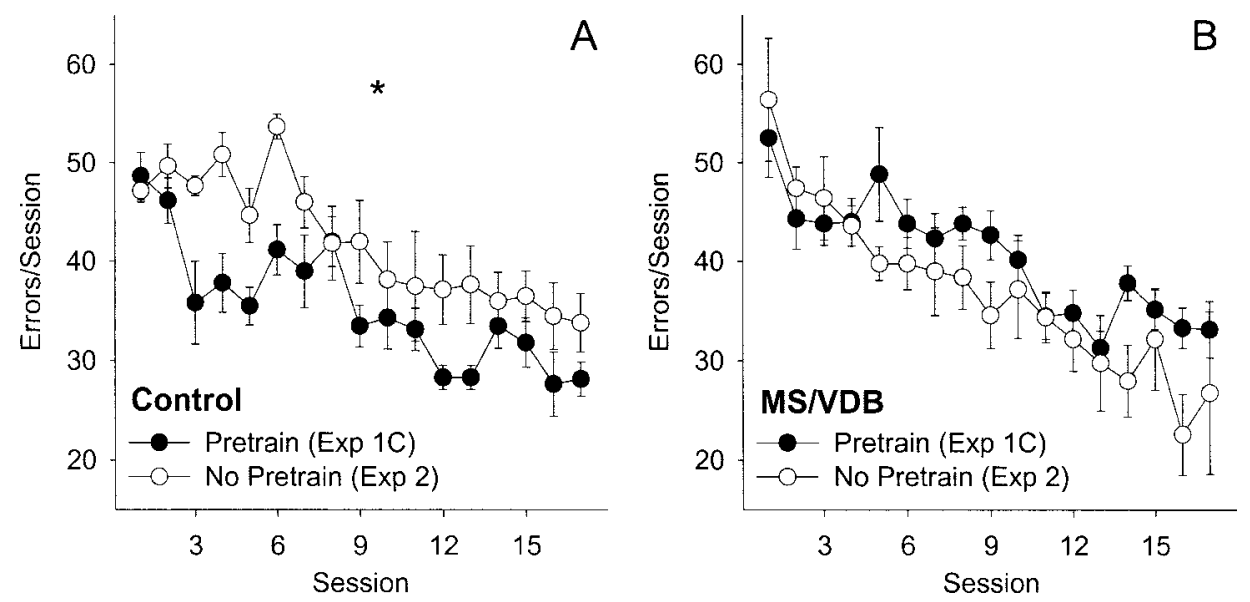

Figure 7. Comparison of Experiments 1C and 2: Central-cue visual-spatial conditional discrimination. Data from acquisition curves on the central-cue visual-spatial discrimination are replotted for the first 17 sessions of rats from both experiments, comparing rats with pretraining on other discrimination problems (Experiment 1C) with those with no pretraining (Experiment 2). A: Control rats with pretraining perform better on the central-cue task than controls without pretraining; there is a significant main effect $(p<.05)$ of pretraining on performance (overall effect indicated by the asterisk). B: Performance on the central-cue task of MS/VDB-lesioned rats with and without pretraining is comparable, although MS/VDB-lesioned rats without pretraining reach criterion significantly faster (see text). Error bars represent \pm 1 SEM. Exp $=$ experiment; MS/VDB = medial septum/ vertical limb of diagonal band.

revealed a significant interaction of lesion and pretraining, $F(1$, $19)=23.93, p<.0005$, and a main effect of lesion, $F(1,19)=$ $5.82, p=.03$, but no main effect of pretraining, $F(1,19)=0.44$, $p=.51$.

To identify the source of these interactions, we performed separate ANOVAs to examine the effects of pretraining in the MS/VDB-lesion and control groups alone. A comparison of acquisition between control rats with or without pretraining on other discriminations revealed a significant difference between these two groups, $F(1,10)=6.09, p=.033$, and a significant Pretraining $\times$ Session interaction, $F(16,160)=1.70, p=.05$ (see Figure 7A). In this instance, controls without pretraining performed more poorly relative to rats with pretraining. Comparison of sessions to criterion in control rats also indicated that pretrained control animals attained criterion in significantly fewer sessions than non-pretrained controls, $F(1,10)=14.95$, $p=.003$. Conversely, there was no significant effect of pretraining on initial acquisition in the MS/VDB-lesioned rats, $F(1,9)=2.12, p=.179$, nor was there a Pretraining $\times$ Session interaction, $F(16,144)=1.06, p=.40$ (see Figure 7B). However, there was an effect of pretraining on sessions to criterion in MS/VDB-lesioned rats, $F(1,9)=10.42, p=.01$; MS/VDB-lesioned rats reached criterion faster when they did not have pretraining on other discrimination problems, in contrast to control rats. This analysis suggests that control rats with pretraining demonstrate faster acquisition early in the task and reach criterion performance levels sooner, whereas pretraining has no such beneficial effect on lesion rats. In fact, MS/VDBlesioned rats may actually experience interference from pretraining on other touchscreen programs because pretrained MS/ VDB-lesioned rats reach criterion later than non-pretrained lesioned rats. All results are summarized in Table 1.

\section{Discussion}

In this study, we sought to explore further the role of cholinergic MS/VDB neurons in visual-spatial conditional tasks, using computer-controlled automated tasks analogous to those used previously in nonhuman primates (Ridley et al., 1999) or in rats (Bussey et al., 1997, 2000). We found that lesions of MS/VDB cholinergic neurons were without effect on simple visual discriminations or on the double-cue visual-spatial conditional problem in which the animal responds to either the left or right object of an identical pair, contingent on which pair of objects was displayed. We found different effects of MS/VDB cholinergic lesions on the central-cue visual-spatial conditional task, depending on whether the rats had been previously trained on other discrimination problems in the touchscreens. In the central-cue visual-spatial conditional problem, rats had to respond to a centrally displayed object and then choose an illuminated panel on either the left or right, contingent on the identity of the object that had been displayed in the center. When rats were tested on a simple visual discrimination and on the double-cue visual-spatial conditional discrimination prior to the central-cue visual-spatial conditional discrimination, control rats performed better than MS/VDB-lesioned rats. In contrast, when the first task the rats encountered was the central-cue visual-spatial conditional discrimination, MS/VDB-lesioned rats performed better than control rats.

The absence of impairment on the double-cue visual-spatial conditional task (Experiment 1B) was unexpected. Because MS/ VDB lesions produced a significant impairment (relative to controls with the same training history) on the central-cue visualspatial conditional task on which the rats were subsequently tested, we conclude that the lack of impairment in the double-cue task was not due to incomplete or behaviorally ineffective lesions. It is possible that rats did not solve this problem conditionally, simply 
Table 1

Summary of Experimental Results

\begin{tabular}{lcc}
\hline \multicolumn{1}{c}{ Experiment or condition } & Initial performance & Sessions to criterion \\
\hline & MS/VDB lesion effects & \\
1A: Simple discrimination & $n s$ & $n s$ \\
1B: Double-cue visual-spatial & $n s$ & $n s$ \\
1C: Central-cue visual-spatial & $p<.05(\mathrm{C}>\mathrm{L})$ & $n s$ \\
2: Central-cue visual-spatial & $n s$ & $p<.05(\mathrm{~L}>\mathrm{C})$ \\
\hline
\end{tabular}

Comparison of transfer effects on central-cue visual-spatial task

Pretrained vs. non-pretrained MS/VDB lesion Pretrained vs. non-pretrained control

$\begin{array}{ll}n s & p<.05 \\ p<.05 & p<.05\end{array}$

Note. $\quad \mathrm{C}>\mathrm{L}$ indicates performance of controls was superior to that of MS/VDB-lesioned rats; $\mathrm{L}>\mathrm{C}$ indicates that performance of MS/VDB-lesioned rats was superior to that of controls. MS/VDB $=$ medial septal/vertical limb of diagonal band.

viewing it as another simple visual discrimination problem. However, because rats found the double-cue task more difficult to solve than the simple visual discrimination presented in Experiment 1A, we are relatively confident that at least the rats were approaching this problem differently than a simple visual discrimination. It is also possible that prior training on a simple visual discrimination somehow protected against an impairment on the double-cue conditional problem. Although we did not test this explicitly, we view this explanation as unlikely, given that monkeys tested on this problem routinely encounter several simple visual discrimination problems before encountering the conditional discrimination and still exhibit impairment on the conditional discrimination (Ridley et al., 1999).

It is also possible that the presentation of the problem in a touchscreen apparatus allowed or encouraged the rats to apply a different learning strategy to the double-cue task. For example, when the problem is presented with real objects on a test tray, animals may approach it differently than when the problem is presented with computer-graphic stimuli. Preliminary experiments in our laboratory, in which rats were tested in double-cue visualspatial conditional learning by examining their approach to real objects separated by several feet in a test arena, suggested that MS/VDB cholinergic lesions produced a severe impairment in conditional learning but not in simple visual discrimination problems presented in the same way (Ghaznavi \& Baxter, 1999). Hence, we were surprised when MS/VDB-lesioned rats solved the double-cue problem in the touchscreen as efficiently as controls. Perhaps the presentation of stimuli on a computer screen encouraged the rats to approach these problems as choosing a rewarded location in a visual scene rather than responding to the two stimuli as individual objects. Notably, fornix lesions (which also disconnect the MS/VDB from the hippocampus) fail to impair the ability of rats to make spatially directed responses on the basis of visual scenes (Gaffan \& Eacott, 1997). Although the previous training of rats on a simple visual discrimination would presumably have encouraged them to respond to the stimuli as individual objects rather than as elements of a scene, future experiments in which the spatial separation and physical characteristics of the stimuli are varied might be able to address this question. Alternatively, the greater spatial separation or three-dimensional characteristics of the real objects in the test arena could have placed a greater burden on spatial processing relative to the analogous task in the touchscreen, but because lesions of MS/VDB cholinergic neurons have repeatedly been shown to have no effect on spatial learning and memory per se (Baxter et al., 1995; Baxter \& Gallagher, 1996; Baxter \& Murg, 2002; Chappell, McMahan, Chiba, \& Gallagher, 1998; McMahan, Sobel, \& Baxter, 1997), this latter possibility seems unlikely. Finally, it is possible that impairments in attentional processing (Baxter et al., 1997, 1999) could have contributed to disrupted performance in a three-dimensional test arena, owing to an inability to reduce attention to task-irrelevant stimuli, although such effects might have been expected to impair simple discriminations as well.

The presence of transfer effects in the control rats that were different from those in the MS/VDB-lesioned rats was surprising to us and unexpected. Several aspects of these data bear discussion. First, rats in our studies generally appeared to find the central-cue visual-spatial conditional problem more difficult to solve than other tasks and than has been reported previously (Bussey et al., 1997, 2000). This may at least partially be a function of the specific instruction stimuli we used for the discrimination problem in our study (T. J. Bussey, personal communication). Second, when rats were tested only on the central-cue task, MS/VDB-lesioned rats performed better than controls. This is similar to the effect of AMPA lesions of the VDB on a similar task (Muir et al., 1996) and has been attributed to a loss of cholinergic input to the anterior cingulate (Bussey et al., 1996; Muir et al., 1996). One hypothesis proposed for this facilitation is that anterior cingulate cortex lesions or loss of cholinergic input to anterior cingulate, may reduce possible disruptive responses to either nonreinforced stimuli or to aversive stimuli (Bussey et al., 1996; Muir et al., 1996).

Third, when rats had prior experience with discrimination tasks in the touchscreen, the lesion effect was reversed, with MS/VDBlesioned rats performing more poorly than control rats with an equivalent training history. This latter effect seemed to be due mostly to the presence of a vast improvement of performance in control rats with pretraining, relative to control rats with no previous experience on different discrimination tasks in the touchscreen. This effect may be due to a general perceptual learning effect in controls that is not seen in MS/VDB-lesioned rats. For example, control rats might learn to pay attention to the shape of 
the object more quickly. However, such an effect might have also been expected to produce a facilitation in learning the double-cue conditional task because of previous experience with the simple discrimination, and no such effect was seen. Alternatively, control rats may generalize their experience with discrimination learning problems to a greater extent than MS/VDB-lesioned rats do. For example, the conditional rule "if shape A go left, if shape B go right" can be applied to both tasks, although the central-cue task requires the more complex spatial response of "go center, then go left" or "go center, then go right." Hence, control rats may be modifying and applying a previously learned strategy. With the aid of such transfer effects, control rats have the ability to learn a subsequent conditional task more efficiently relative to MS/VDBlesioned rats. This may be qualitatively similar to impairments in flexibility of memory expression seen after medial temporal lobe damage (Reber, Knowlton, \& Squire, 1996) as well as impairments in transfer of learning about specific discrimination problems in aged humans with hippocampal atrophy (Myers et al., 2002). It is interesting to note that a related finding has been observed when response rules in a vigilance task are reversed; rats with MS/VDB cholinergic lesions are facilitated in learning the reversed rules (Sarter, Draut, Herzog, \& Bruno, 2002). This may represent an instance in which failing to transfer learning to a new test situation actually benefits MS/VDB-lesioned rats because the previously learned rules do not interfere with learning the new (reversed) response rules.

In the present study, MS/VDB-lesioned rats could perform comparably to, or even better than, control rats on visual-spatial conditional learning tasks. However, they failed to benefit from positive transfer effects from previous experience in discrimination learning and even showed some evidence of negative transfer from previous training. Taken together, our results (along with those from a previous study; Janisiewicz et al., in press) suggest that MS/VDB cholinergic neurons play a role in relating or modifying learned rules in memory rather than in conditional learning per se. This extends the observation of Ridley and Baker (1997) that disruption of the septohippocampal system produces impairment in a particular aspect of information processing. It seems that this aspect of septohippocampal function specifically requires intact septal cholinergic neurons. However, we suggest that this impairment is not necessarily related to difficulty in placing information into long-term memory, as posited by Ridley and Baker, on the basis of other experiments that have found no disruption in long-term recall of associative learning after lesions of MS/VDB cholinergic neurons (e.g., Vale-Martínez et al., 2002). Instead, we suggest that MS/VDB cholinergic neurons are specifically involved in application of learned behavioral rules or generalization of previous behavioral experience to novel test situations. In the absence of these neurons, the ability to apply such experience to regulate learning of new behavioral rules may be disrupted.

Finally, we note that if we considered the findings of Experiment 1 and Experiment 2 separately, two opposite conclusions about the role of MS/VDB cholinergic neurons in visual-spatial conditional learning would be drawn. We suggest that rather than providing a confound in behavioral neuroscience experiments, examination of rats with lesions of MS/VDB cholinergic neurons (or disruption of any other neural system) in multiple behavioral tasks, tested sequentially, may provide an interesting system in which to examine the neural substrates of transfer of learning and generalization of behavioral experience.

\section{References}

Baxter, M. G., Bucci, D. J., Gorman, L. K., Wiley, R. G., \& Gallagher, M. (1995). Selective immunotoxic lesions of basal forebrain cholinergic cells: Effects on learning and memory in rats. Behavioral Neuroscience, 109, 714-722.

Baxter, M. G., \& Chiba, A. A. (1999). Cognitive functions of the basal forebrain. Current Opinion in Neurobiology, 9, 178-183.

Baxter, M. G., \& Gallagher, M. (1996). Intact spatial learning in both young and aged rats following selective removal of hippocampal cholinergic input. Behavioral Neuroscience, 110, 460-467.

Baxter, M. G., Gallagher, M., \& Holland, P. C. (1999). Blocking can occur without losses in attention in rats with selective lesions of hippocampal cholinergic input. Behavioral Neuroscience, 113, 881-890.

Baxter, M. G., Holland, P. C., \& Gallagher, M. (1997). Disruption of decrements in conditioned stimulus processing by selective removal of hippocampal cholinergic input. Journal of Neuroscience, 17, 52305236.

Baxter, M. G., \& Murg, S. L. (2002). The basal forebrain cholinergic system and memory: Beware of dogma. In L. R. Squire \& D. L. Schacter (Eds.), Neuropsychology of memory (3rd ed., pp. 425-436). New York: Guilford Press.

Berger-Sweeney, J., Stearns, N. A., Frick, K. M., Beard, B., \& Baxter, M. G. (2000). Cholinergic basal forebrain is critical for social transmission of food preferences. Hippocampus, 10, 729-738.

Bussey, T. J., Duck, J., Muir, J. L., \& Aggleton, J. P. (2000). Distinct patterns of behavioural impairments resulting from fornix transection or neurotoxic lesions of the perirhinal and postrhinal cortices in the rat. Behavioural Brain Research, 111, 187-202.

Bussey, T. J., Muir, J. L., Everitt, B. J., \& Robbins, T. W. (1996). Dissociable effects of anterior and posterior cingulate cortex lesions on the acquisition of a conditional visual discrimination: Facilitation of early learning vs. impairment of late learning. Behavioural Brain Research, 82, 45-56.

Bussey, T. J., Muir, J. L., Everitt, B. J., \& Robbins, T. W. (1997). Triple dissociation of anterior cingulate, posterior cingulate, and medial frontal cortices on visual discrimination tasks using a touchscreen testing procedure for the rat. Behavioral Neuroscience, 111, 920-936.

Bussey, T. J., Muir, J. L., \& Robbins, T. W. (1994). A novel automated touchscreen procedure for assessing learning in the rat using computer graphic stimuli. Neuroscience Research Communications, 15, 103-110.

Cahill, J. F. X., \& Baxter, M. G. (2001). Cholinergic and noncholinergic septal neurons modulate strategy selection in spatial learning. European Journal of Neuroscience, 14, 1856-1864.

Chappell, J., McMahan, R., Chiba, A., \& Gallagher, M. (1998). A reexamination of the role of basal forebrain cholinergic neurons in spatial working memory. Neuropharmacology, 37, 481-487.

Chiba, A. A., Bucci, D. J., Holland, P. C., \& Gallagher, M. (1995). Basal forebrain cholinergic lesions disrupt increments but not decrements in conditioned stimulus processing. Journal of Neuroscience, 15, 73157322.

Freund, T. F. (1989). GABAergic septohippocampal neurons contain parvalbumin. Brain Research, 478, 375-381.

Gaffan, E. A., \& Eacott, M. J. (1997). Spatial memory impairment in rats with fornix transection is not accompanied by a simple encoding deficit for directions of objects in visual space. Behavioral Neuroscience, 111, 937-954.

Ghaznavi, S., \& Baxter, M. G. (1999). [Conditional visual-spatial discrimination learning with 3-dimensional objects in rats with cholinergic lesions of medial septum/vertical limb of the diagonal band.] Unpublished raw data.

Janisiewicz, A. M., Jackson, O., III, Firoz, E. F., \& Baxter, M. G. (in press). Environment-spatial conditional learning in rats with selective lesions of medial septal cholinergic neurons. Hippocampus.

Linster, C., Garcia, P. A., Hasselmo, M. E., \& Baxter, M. G. (2001), Selective loss of cholinergic neurons projecting to the olfactory system 
increases perceptual generalization between similar, but not dissimilar, odorants. Behavioral Neuroscience, 115, 826-833.

Marston, H. M., Everitt, B. J., \& Robbins, T. W. (1993). Comparative effects of excitotoxic lesions of the hippocampus and septum/diagonal band on conditional visual discrimination and spatial learning. Neuropsychologia, 31, 1099-1118.

McGaughy, J., Dalley, J. W., Morrison, C. H., Everitt, B. J., \& Robbins, T. W. (2002). Selective behavioral and neurochemical effects of cholinergic lesions produced by intrabasalis infusions of 192 IgG-saporin on attentional performance in a five-choice serial reaction time task. Journal of Neuroscience, 22, 1905-1913.

McGaughy, J., Kaiser, T., \& Sarter, M. (1996). Behavioral vigilance following infusions of $192 \mathrm{IgG}$-saporin into the basal forebrain: Selectivity of the behavioral impairment and relation to cortical AChEpositive fiber density. Behavioral Neuroscience, 110, 247-265.

McMahan, R. W., Sobel, T. J., \& Baxter, M. G. (1997). Selective immunolesions of hippocampal cholinergic input fail to impair spatial working memory. Hippocampus, 7, 130-136.

Muir, J. L., Bussey, T. J., Everitt, B. J., \& Robbins, T. W. (1996). Dissociable effects of AMPA-induced lesions of the vertical limb of the diagonal band of Broca on performance of the 5-choice serial reaction time task and on acquisition of a conditional visual discrimination. Behavioural Brain Research, 82, 31-44.

Myers, C. E., Kluger, A., Golomb, J., Ferris, S., de Leon, M. J., Schnirman, G., \& Gluck, M. A. (2002). Hippocampal atrophy disrupts transfer generalization in nondemented elderly. Journal of Geriatric Psychiatry and Neurology, 15, 82-90.
Reber, P. J., Knowlton, B. J., \& Squire, L. R. (1996). Dissociable properties of memory systems: Differences in the flexibility of declarative and nondeclarative knowledge. Behavioral Neuroscience, 110, 861-871.

Ridley, R. M., \& Baker, H. F. (1997). Evidence for a specific information processing deficit in monkeys with lesions of the septo-hippocampal system. Cortex, 33, 167-176.

Ridley, R. M., Barefoot, H. C., Maclean, C. J., Pugh, P., \& Baker, H. F. (1999). Different effects on learning ability after injection of the cholinergic immunotoxin ME20.4IgG-saporin into the diagonal band of Broca, basal nucleus of Meynert, or both in monkeys. Behavioral Neuroscience, 113, 303-315.

Sarter, M., Draut, A., Herzog, C. D., \& Bruno, J. P. (2002). Effects of septohippocampal cholinergic deafferentation on attention and learning. Society for Neuroscience Abstracts, 28, 8.

Vale-Martínez, A. M., Baxter, M. G., \& Eichenbaum, H. (2002). Selective lesions of basal forebrain cholinergic neurons produce anterograde and retrograde deficits in a social transmission of food preference task in rats. European Journal of Neuroscience, 16, 983-998.

Waite, J. J., Wardlow, M. L., \& Power, A. E. (1999). Deficit in selective and divided attention associated with cholinergic basal forebrain immunotoxic lesion produced by 192-saporin; motoric/sensory deficit associated with Purkinje cell immunotoxic lesion produced by OX7-saporin. Neurobiology of Learning and Memory, 71, 325-352.

Received March 14, 2003

Revision received May 26, 2003

Accepted June 9, 2003

\section{Wanted: Your Old Issues!}

As APA continues its efforts to digitize journal issues for the PsycARTICLES database, we are finding that older issues are increasingly unavailable in our inventory. We are turning to our long-time subscribers for assistance. If you would like to donate any back issues toward this effort (preceding 1982), please get in touch with us at journals@apa.org and specify the journal titles, volumes, and issue numbers that you would like us to take off your hands. 